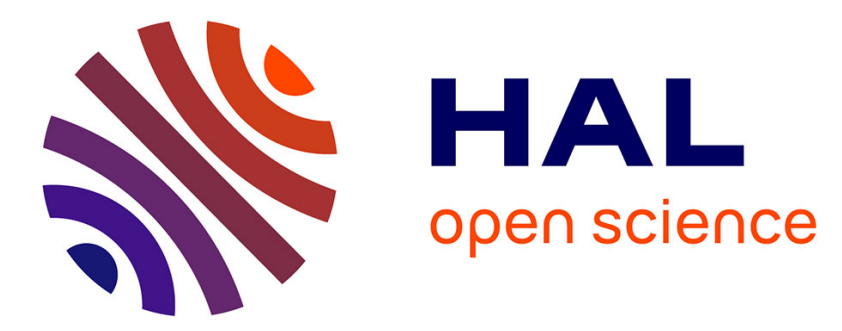

\title{
Répertoires juridiques et affirmation identitaire Baudouin Dupret
}

\section{To cite this version:}

Baudouin Dupret. Répertoires juridiques et affirmation identitaire. Droit et Société, 1996, 34, pp.591611. hal-02160899

\section{HAL Id: hal-02160899 \\ https://hal.science/hal-02160899}

Submitted on 19 Sep 2019

HAL is a multi-disciplinary open access archive for the deposit and dissemination of scientific research documents, whether they are published or not. The documents may come from teaching and research institutions in France or abroad, or from public or private research centers.
L'archive ouverte pluridisciplinaire HAL, est destinée au dépôt et à la diffusion de documents scientifiques de niveau recherche, publiés ou non, émanant des établissements d'enseignement et de recherche français ou étrangers, des laboratoires publics ou privés. 


\section{Répertoires juridiques et affirmation identitaire}

Baudouin DUPRET

Au départ d'un corpus d'entretiens constitué sur notre terrain d'enquête, l'Égypte contemporaine, on voudrait s'attacher au rôle pivot de la problématique identitaire dans la question du droit et du discours portant sur ses différents répertoires, en l'espèce ce que l'on appelle les répertoires juridiques positif et islamique (qu'on entend généralement sous le vocable de sharî'a).

Elever le droit au rang de problématique identitaire n'est pas sans présenter d'importants écueils. Non seulement la question est-elle galvaudée par le recours trop systématique qui y est fait, mais, surtout, il y a lieu de constater à quel point on évite rarement le piège de substantialisation qu'elle tend. Sur ce point, l'approche par les représentations sociales fournit probablement une clé d'entrée digne d'intérêt. Il ne s'agit pas de donner consistance à une prétention identitaire, en lui trouvant des arguments d'ordre historique, géographique, pyschologique, culturel ou autre. Tout au contraire, l'idée consiste à montrer à quel point l'identité est un concept systématiquement transformé au travers des processus cognitifs individuel et social. L'identité au soi individuel ou social, que postule la prétention identitaire, ne se laisse appréhender qu'à la seule condition d'y voir le produit d'une représentation socialement construite que l'individu a de lui-même et du groupe auquel il prétend appartenir. Cette typification du soi (individuel ou collectif) est à la fois produite par la représentation que le groupe se fait de son être constitutif et productrice de nouvelles représentations intégrant à des degrés divers l'appréhension toujours mouvante d'un réel toujours changeant.

Après avoir très brièvement rappelé la nature proprement communicationnelle du droit, on s'attachera, en s'appuyant essentiellement sur les travaux de Carol Greenhouse, Boaventura De Sousa Santos et Massaji Chiba, à construire une démonstration articulée autour de trois points: la nature classificatoire du droit en tant que système symbolique ; la constitution d'espaces comme résultante de ce complexe d'assignations catégorielles ; l'établissement de frontières définissant une altérité et une identité.

\section{Droit et communication}

Analyser le droit par le biais du concept de représentations sociales, c'est reconnaître le caractère intrinsèquement individuel et social de sa construction. Certainement irréductibles l'un à l'autre, ces deux niveaux ne doivent pas moins nécessairement être couplés. On peut en déduire la nature intrinsèquement communicationnelle du droit. Habermas, au départ d'un critère d'intersubjectivité, développe un modèle mettant aux prises des individus qui, en communiquant, visent à obtenir de leur interlocuteur la validation de leur énoncé. Celle-ci peut être de différents types et, en fonction de la situation, le locuteur adoptera l'énoncé lui paraissant le plus apte à répondre à la relation au monde qu'elle implique et exigera que cet énoncé reçoive la validation qui lui correspond. Il cherchera donc à adopter un type adéquat de rationalité — de répertoire aurions-nous tendance à dire. La question de la rationalité se réfère donc à un contexte systématique de prétention à la validité ${ }^{1}$.

1 Melkevik B. 1990, «Le modèle communicationnel en science juridique : Habermas et le droit». Les Cahiers de Droit. 31/3. 
S'agissant du discours juridique, une approche dialectique des niveaux individuel (les différentes cognitions) et social (processus communicationnel) permet de relever que c'est aussi bien le sujet qui l'agence que les conditions sociales qui suscitent et assurent son déploiement ${ }^{2}$. S'il fallait adresser une critique à la théorie habermassienne, elle devrait donc porter, à notre sens, sur la nature hypostasiée de la communication. Le modèle communicationnel d'Habermas est, en effet, de toute apparence, un modèle de communication parfaite, une sorte de pendant philosophique au célèbre postulat microéconomique. Or, la communication n'est jamais parfaite. En conséquence, on aura plutôt tandance à considérer que l'acteur-sujet-individu cherche à compenser les errements de la communication par une projection des réponses qu'il devrait à ses yeux obtenir en situation de communication parfaite. D'où l'importance majeure d'une analyse en termes d'anticipations des résultats que le choix d'une rationalité peut donner, en termes de recherche de normalité3.

Le droit est donc communication, lieu et objet de communication. L'étude du droit n'est plus, depuis longtemps, la seule étude des modes de résolution des conflits. Pourtant, même réduite à cela, l'étude du droit acquiert une dimension nouvelle si l'on adopte la perspective communicationnelle. Les conflits eux-mêmes peuvent en effet être abordés en tant que communications, c'est-à-dire en tant que dialogues consistant en «l'échange public d'affirmations («claims») et contre-affirmations («counterclaims») normatives $»^{4}$. Ceci conduit à remarquer que le droit, en tant qu'il est communication, constitue un langage et que parler de langage mène à adopter une double perspective : d'une part, la communication par le langage est un processus d'échanges; d'autre part, le langage est le produit réactualisé d'un fond commun, d'un espace culturel et identaire.

Il n'est qu'à lire Raymond Verdier pour savoir que le droit constitue «un système de communication et d'échange de valeurs instaurant des relations symboliques entre les membres (individus et groupes) d'une même unité politique ou de différentes unités appartenant à un groupement politique plus vaste» 5 . Sa vocation consiste bien à modaliser la communication entre les individus et les groupes en désignant ses acteurs, son champ et ses moyens ${ }^{6}$. Si l'on adopte une perspective interactionniste, on dira que cette désignation procède des significations que les individus assignent aux «choses» (objets, êtres humains, institutions, valeurs, situations, ...). Ces significations, qui sont le produit des interactions individuelles, ont une portée symbolique ${ }^{7}$. En droit, les interactions (et la communication qu'elles supposent) procèdent ainsi à des étiquetages, à des typifications, à des qualifications. Ces qualifications s'inscrivent dans le support communicationnel et interactionniste du groupe social qu'elles concernent et font l'objet de réajustements constants (processus interprétatif). On peut donc parler du droit comme d'un système symbolique, dont le propre est d'assurer une représentation

2 Vignaux G. 1979, «Argumentation et discours de la norme». Langages: 53: 69.

3 Dupret B. 1995, «Le juge et le jeu de la normalisation islamique du droit positif». Droit et Cultures. 30 .

$4 \quad$ Greenhouse C. J. 1982, «Looking at culture, looking for rules». Man. 17: 70

5 Verdier R. 1981, «Premières orientations pour une anthropologie du droit». Droit et

Cultures. 1: 20

$6 \quad$ Id.: 13 .

$7 \quad$ Winkin Y. 1993, v «Interactionnisme symbolique». In Arnaud A.-J. (sous la dir.),

Dictionnaire encyclopédique de théorie et de sociologie du droit. Paris: LGDJ. 
signifiante du réel permettant l'action sociale des individus ${ }^{8}$. Cette médiation est structurée, en ce sens qu'elle est déterminée par les structures sociales (dont elle s'autonomise partiellement - autopoièse relative), et structurante, dans la mesure où une situation, dès lors qu'elle est conçue comme réelle, exerce en tant que telle, dans ses conséquences, une influence sur le réel. Elle est structurée parce qu'elle se fonde largement sur un réservoir de connaissances accumulées, mais elle est aussi structurante parce que relativement autonome.

\section{Droit et classification}

Tout système juridique semble devoir se caractériser par l'adoption d'un système classificatoire ou catégoriel propre : «le droit est un processus, sans cesse renouvelé, de classification du réel»» ${ }^{9}$. Confrontés à un objet, les individus, par leur interaction, sont conduits à étiqueter, typifier, qualifier cet objet, et toute nouvelle confrontation est source d'ajustement des schèmes catégoriels et classificatoires existants. La connaissance d'un objet procède donc des principes propres aux structures sociales dans lesquelles s'inscrit l'interaction. Ces principes, qu'on peut appeler règles culturelles («cultural rules» $\left.{ }^{10}\right)$, ne constituent pas tant des règles prescriptives de conduite qu'une forme de savoir ${ }^{11}$. Ceci vient conforter l'idée que les transactions (ou interactions) sont à la fois structurées (par les règles qui les précèdent) et structurantes (elles réajustent constamment ces règles). Il devient difficile, dans ce contexte, de maintenir une attitude positiviste tendant à substantialiser les règles culturelles. La perspective transactionniste, parce qu'elle traite de la structure sociale comme d'un savoir, réagit contre la conception positiviste des normes et la remplace par une conception des normes entendues comme «présuppositions» («assumptions»12), «significations» $\left(\ll\right.$ meanings $\left.{ }^{13}\right)$ ou encore «instruments d'évaluation» ${ }^{14}$.

Les normes deviennent alors des modèles de reconstruction de la réalité qui s'insèrent dans des processus de régularisation et d'ajustement situationnel couplés à un fort facteur d'indétermination ${ }^{15}$. Les normes ont pour fonction de figer la vie sociale et d'ainsi la faire échapper à l'indétermination, tout en étant incapables d'y parvenir complètement ${ }^{16}$. Mais alors, dans tout discours où il y est fait recours, la norme devient plus une rationalisation qu'une motivation : elle est de l'ordre de la justification. Etudier

$8 \quad$ Crespi F. 1993, v «Action (sociologie de l')». In Arnaud A.-J., op. cit.

9 Assier-Andrieu L. 1987, «Le juridique des anthropologues». Droit et Société. 5: 27.

10 Salisbury R. F. 1976, «Transactions or transactors ? An economic anthropologist's view». In Kapferer B. (ed.), Transaction and meaning. Philadelphia: Institute for the Study of Human Issues, 47, cité par Greenhouse op. cit.: 68.

11 C. Greenhouse, op. cit.: 68. Ce qui fait justement dire à Cohen et Comaroff («The management of meaning : on the phenomenology of political transactions». In Kapferer B. (ed.), Transaction and meaning. Philadelphia: Institute for the Study of Human Issues, 95) : «la manipulation du savoir («"la gestion du sens") devient un "aspect essentiel du pouvoir"».

12 Kapferer, op. cit.

13 Cohen and Comaroff, op. cit.

14 Paine R. 1976, «Two modes of exchange and mediation». In Kapferer, op. cit

15 Moore S. F. 1978, Law as Process : An Anthropological Approach. London, Henley and

Boston, Routledge \& Kegan Paul, 39.

16 Id.: 41. 
la norme consiste de ce fait davantage à analyser un répertoire d'argumentation qu'une structure de motivation ${ }^{17}$. La justification suppose la connaissance de la structure sociale en même temps qu'elle procède des représentations que le locuteur se fait de luimême, de la situation, de son auditoire et de leurs relations. On voit ce qui distingue la norme de la règle. La norme est invoquée par l'individu qui l'impute à la société, alors que la règle est promulguée publiquement par une autorité ; la norme est la résultante d'une expérience commune, d'une culture, alors que la règle est produite par la décision d'une autorité ; on n'est pas explicitement tenu d'être en conformité avec la norme, alors qu'on est tenu d'être en règle ; la norme s'expose alors que la règle impose ; la norme est un adverbe (une modalité) alors que la règle est un nom (un énoncé performatif). Le propre de la norme est la classification sociale. «La pensée normative requiert de celui qui pense qu'il établisse une analogie entre sa situation et les justifications invoquées par quelqu'un d'autre. C'est donc un processus de classification et de reclassification de soi-même par rapport à tout ou partie de la société environnante. Le résultat de la pensée normative est un système d'inclusions et d'exclusions» ${ }^{18}$. Là où la règle est un élément prescriptif ou proscriptif d'actes ou de groupes d'actes particuliers, la norme est un élément classificateur des actes en catégories de normal et d'anormal. La norme est donc justification. Faire référence à une norme revient à se justifier au regard des autres et en opposition aux autres sur fond d'une expérience commune avec laquelle on établit une analogie. La justification sera d'autant plus opératoire qu'elle procèdera d'une appréhension exacte de ce qui est reconnu socialement. En ce sens, les schèmes catégoriels et classificatoires normatifs opéreront d'autant mieux qu'ils seront liés aux classifications sociales - ce que, par commodité, on appelle culture ${ }^{19}$.

Si l'on en vient aux entretiens qu'on a pu conduire avec différents professionnels égyptiens du droit, on remarquera que c'est presque systématiquement que les interlocuteurs font référence à des schèmes classificatoires producteurs de normes (plus que de règles). Vision agissante du réel, le droit l'est assurément à entendre ces discours,

17 Bailey F. G. 1973, Debate and Compromise : The politics of innovation. Totowa: Rowman \& Littlefield, 326, cité par Greenhouse, op. cit.

18 Greenhouse, op. cit.: 65.

$19 I d .: 71$. Sauf à convenir que le droit n'est que ce qui se présente comme tel, il faut admettre que l'étude du droit - au moins du point de vue critique, sociologique et anthropologique - dépasse la question de la règle de droit pour s'étendre à celle de la norme. L'objet d'étude consiste alors dans les images de la structure sociale et le langage produisant et véhiculant un savoir et un sens normatifs. La tâche de l'anthropologue du droit consiste, dans cette hypothèse et à suivre Carol Greenhouse, à «chercher les systèmes de justifications impliquant le sens de l'ordre (social) à des lieux et moments particuliers - en d'autres mots, la culture» (id.: 71). Il faut s'interroger sur ces ordres normatifs inhérents à la dynamique des savoirs socio-culturels (Assier-Andrieu , op. cit.:: 96) et cette interrogation dépasse le cadre du seul droit auto-proclamé. Les notions d'ordre social et de reproduction sociale sont matière à une anthropologie du normatif, c'est-à-dire de la norme juridique, politique, religieuse, économique, etc. Est-ce à dire que «l'aboutissement de ce type de raisonnement, explicitement détaché de l'emprise de la pensée juridique, est une non moins explicite négation de la spécificité juridique du domaine abordé, et donc de la licéité qu'il y aurait à qualifier de juridique une anthropologie dont l'objet se dérobe» (id.: 99) ? Non, à notre sens, dès lors qu'on admet tout simplement qu'une anthropologie du droit auto-proclamé et de ses institutions garde toute sa raison d'être en tant qu'objet et lieu, parmi d'autres, d'une anthropologie plus générale du normatif. 
et les répertoires auxquels il est recouru traduisent un choix partiellement préjugé qu'il faut nécessairement resituer dans le contexte d'une «culture» dont le droit est un élément, voire un instrument de mise en visibilité. Les extraits suivant devraient permettre de s'en convaincre.

«Le confinement de l'islam dans nombre de législations est un simplisme et un dépouillement de son contenu véritable. C'est pourquoi je ne peux pas dire que le droit des procédures ou le droit commercial ou le droit international, que toutes ces choses ne sont pas, dans leurs grandes lignes, véritablement organisées par l'islam. Mais en parler simplement en disant que nous voulons que ces lois soient en conformité avec l'islam est simpliste, dans la mesure où, même si nous imposons l'application des peines légales $(h u d \hat{u} d$ ), celui qui appliquera ces peines sera lui-même un juge (qâdî) requérant l'application de ces peines pour les faire entrer en vigueur. (...)

«Effectivement, l'Occident ne voit que la question des mains coupées, etc. (...) Mais en fait, la société musulmane n'applique pas ces peines. Elles ont été instaurées pour ne pas être appliquées. La société musulmane ne commet pas les crimes qui justifient l'application des peines Légales. Pas d'application de la peine prévue pour la fornication, à moins que quatre personnes n'aient vu l'acte être perpétré. Le droit positif condamne pour fornication sur la base de présomptions. De plus, il n'y a aucune ignominie dans ces peines Légales. Elles sont très raisonnables. En islam, c'est la société qu'on condamne dans la mesure où elle a laissé le voleur voler. Les peines Légales sont assorties de conditions, en dépit du fait que l'Occident s'est emparé de la question de ces peines pour s'en prendre au mouvement islamique» (entretien avec NH, avocat, janvier 1994).

«Les codifications peuvent s'avérer utile, en droit administratif par exemple. Cela étant, le fiqh, le Coran et la Sunna sont comme une Constitution. Ils contiennent les principes généraux que les codifications ne peuvent pas contredire. (...)

«La sharî‘ $a$ islamique englobe le fiqh. Mais si, par sharî‘ $a$ islamique, on entend le Livre et la Sunna, nous dirons que le Livre, ce sont des règles générales qui nécessitent une exégèse. Quant au fiqh, il est divisé, comme l'ont fait les jurisconsultes, en traditions explicatives (shâriha), complétives (mukammila) et interprétatives (mufassira). Il y a aussi des traditions orales (qawliyya), pratiques (fi liyya) et décisionnelles (taqrîriyya). La tradition explicative porte sur un verset obscur, dont le libellé n'est pas courant. Le Prophète l'a alors expliqué, comme dans le verset «Les femmes divorcées doivent attendre un délai de trois qurû'»(Coran II, 228), qui signifie que les femmes divorcées, une fois divorcées de leur mari, ne se marieront pas avant l'écoulement de la période viduité qui est de trois qurû́. Les gens ne connaissent pas le sens du terme «qurû́'». S'agit-il de trois indispostions féminines (haydât) ou de trois périodes sans indisposition (tuhûr min al-hayda). La Tradition interprète ce propos. Il s'agit alors d'une tradition complétive. Quand une règle précise est révélée dans le Coran comme, par exemple : «Il vous est interdit la chair d'une bête morte, le sang, la viande de porc et sur quoi on invoqué un autre que Dieu» (Coran II, 173). Le sang est interdit, mais quel sang ? Le Prophète a établi, dans une tradition explicative, qu'il s'agit du sang qui a été versé (masfûh). Dieu - gloire à Lui ! - a ainsi mentionné dans une série de versets que la prière et l'aumône étaient obligatoires pour tous. La Tradition prophétique nous a expliqué les modalités de la prière et de l'aumône. La sharî‘ $a$ islamique a donc été révélée pour interpréter ces principes.

«Il n'y a pas de choix sur les propos du Coran et de la Sunna, mais les propos fiqh font l'objet de jugements de préférence (marâjih). Il faut absolument oeuvrer à l'aide des textes du Coran et de la Sunna, mais les propos tenus et les règles établies par les jurisconsultes peuvent être contredits ou mis en oeuvre, dans la mesure où l'exégèse d'une personne ou d'un jurisconsulte a pu être influencée par la situation qui prévalait dans son pays. (...)

«Le droit et l'équité sont les deux axes principaux autour desquels tourne la sharî‘ $a$ islamique. Celui qui ne respecte ni l'un ni l'autre n'entre pas dans le champ de la sharî‘ $a$ islamique» (entretien avec MN, avocat, janvier 1994).

Il reste qu'on peut se demander dans quelle mesure les différents répertoires juridiques utilisés par les acteurs interrogés ne constituent pas les différents niveaux d'un discours de justification (caractère normatif du répertoire juridique islamique) sur un système de droit (caractère régulatoire du répertoire juridique positif). En ce sens, il n'y aurait pas conflictualité de différents systèmes de droit ou de différents répertoires 
juridiques, mais bien tension opposant les différents cercles de la validité des règles de droit ${ }^{20}$. Le discours des professionnels interrogés pourrait alors s'analyser en stratégies diverses de réduction de cette tension visant à faire correspondre au cercle de la légalité (règles de droit positif) le cercle de l'effectivité (respect du droit positif existant) et, surtout, le cercle de la légitimité (inscription de la règle dans les schèmes classificatoires de la «culture» normative égyptienne).

La norme procède davantage d'une classification du réel opérant a posteriori comme une justification, là où la règle (de droit) procède plutôt comme une interprétation prescriptive et a priori de ce même réel. Si l'on accepte, par ailleurs, le point du raisonnement par lequel on situe la norme islamique à l'intérieur du cercle de légitimité et la règle de droit positif à l'intérieur du cercle de légalité, on fera alors le constat d'un répertoire islamique consistant essentiellement en une forme de savoir, plus proche généralement du sens commun que d'un savoir technique, qu'il est difficile de substantialiser (provisoirement au moins) et qui fonctionne sur le mode de la présupposition, de la signification ou de l'instrument d'évaluation. De ceci découle très naturellement le caractère à la fois général et très largement indéterminé du référent islamique, quand bien même le fait d'y recourir répond à une volonté de fixation d'une réalité changeante.

«Il ne faut pas que les autres croient que nous vivons, en tant que pays islamique qui applique le droit positif, toujours derrière les innovations auxquelles procèdent les autres régimes, et particulièrement les régimes occidentaux développés, dans leurs législations. Chaque pays a son idéologie propre et des racines qui plongent dans son histoire et se répercutent sur son présent : le passé se prolonge dans le présent et aussi dans le futur. (...) On peut dire, au sujet des infractions pénales, que que les règles stipulées par le droit pénal égyptien sont conformes, dans leur contenu, aux peines discrétionnaires (jarâ'im al-ta 'zîr) de la sharî'a islamique et sont pour partie conformes aux peines Légales et pour partie non conformes. On peut donc affirmer que la $\operatorname{shar}^{\prime} a$ islamique a très largement investi le droit positif égyptien. (...) La revendication d'une application de la sharî‘ $a$ en Égypte porte sur les quelques dispositions qui ne sont pas appliquées et je pense que c'est une revendication qui correspond à notre régime général en Égypte, sans doute ni ambiguïté. (...)

«Je suis naturellement partisan de l'application de la $\operatorname{sharî‘}^{`} a$ islamique. Il y a trois maillons (halaqât) dans la vie de l'homme. Le premier maillon, c'est le lien qui unit l'homme à son Seigneur $(r a b b)$; le second, celui qui l'unit à lui-même ; le troisième, celui qui l'unit à autrui. En droit positif, il n'y a pas de loi qui régisse la relation de l'homme à son Seigneur. Avez-vous une loi qui vous condamne à la prison si vous n'allez pas à l'église ? (...) Non, mais vous avez des lois qui régissent votre relation à autrui. Dans la sharî‘ $a$ islamique, on trouve l'ensemble de ces maillons. Le maillon qui régit la relation de l'homme à son Seigneur, c'est celui qui lui ordonne de suivre une doctrine (minhaj) déterminée. Je sais de ce fait que Dieu me demandera des comptes dans l'au-delà : il faut donc que ma relation à mon Seigneur soit forte. Je dois prier, jeûner, faire l'aumône, faire le pélerinage dans la mesure du possible et observer l'ensemble de la doctrine islamique. En cas de manquement, je sais que Dieu me pénalisera. Le maillon qui m'unit à moi-même consiste à ce que je me protège et que je n'aille pas à ma perte, Dieu pourrait m'en demander des comptes. Enfin, la relation à autrui, dans le cadre de la sharî́ $a$ islamique, est fondée sur la sharî́' $a$ et ne peut être contraire, ni en tout ni en partie, aux deux précédentes. Dans le cadre du droit positif, il n'y a pas de loi réglementant ma prière ou mon suicide, etc. Dans le cadre du droit positif, il n'est pas de loi sachant que je prie ou non, que je jeûne ou non, que je vais me suicider ou non, etc., mais il y a d'autres lois qui réglementent les transactions que je passe avec autrui. Si nous nous occupons de ce maillon, nous constatons que la $\operatorname{sharî́}^{\prime} a$ islamique, quand elle statue sur la relation de quelqu'un avec autrui, est plus précise, plus englobante, plus générale et plus équitable que les dispositions que le droit positif peut établir. Le droit islamique réglemente donc ce que ne réglemente pas le droit positif, à savoir la relation à soi-même et à son Seigneur. Pourquoi ? Parce que moi, tant que la barrière (wâzi ${ }^{\circ}$ de la religion me fait

20 Ost F. 1987, «Essai de définition et de caractérisation de la validité juridique». In Rigaux F. et Haarscher G. (sous la dir.), Droit et pouvoir. t. I - La validité. Bruxelles: Story-Scientia 
obstacle, je crains Dieu en toute chose. Je vous ai dit qu'il y avait des lois égyptiennes conformes à la sharî' $a$ islamique et d'autres, non. Si la sharî'a était appliquée correctement (salîman) ou complètement (kâmilan), vous constateriez que cette barrière serait plus importante : en tant que musulman, il me serait fait obligation d'avoir de bonnes relations avec autrui, de ne m'en prendre à personne, de ne pas usurper le bien d'autrui, de me préserver moi-même, parce que, du point de vue religieux, il existe un compte à rendre dans l'autre monde auquel je dois croire» (entretien avec MB, avocat, novembre 1993 et janvier 1994).

«Naturellement. La finalité de la sharî'a, c'est l'intérêt (maslaha) des croyants ('ubbâd) et des hommes (bashar). Les jurisconsultes ont défini cet intérêt. Dans un livre comme les muwâfaqât d'alShâtibî, il est question des objectifs (maqâsid) de la sharî'a. Il en a défini cinq : l'intérêt de la religion (dîn), l'intérêt relatif à l'intégrité physique (muhâfaza 'alâ al-nafs), celui relatif à l'argent (mâl), à la raison ('aql) et à la progéniture (nasab). Ibn al-Qayyim, dans son Siyâsa shar iyya (Politique Légale) parle également des intérêts. La Loi (shar') est fondamentalement liée à l'intérêt. Il a ailleurs parlé des divergences qui sont fonction du temps et du lieu, dans sept circonstances. Il donne ainsi l'exemple de quelqu'un qui avait bu du vin et qui devait donc être puni pour cela, mais qui y échappe parce qu'on est en situation de guerre. Il y a beaucoup d'exemples de ce type. Il y a ainsi un proverbe qu'on apprend dans les grands traités : «quand il y a un intérêt, il y a la Loi de Dieu» (entretien avec BI, magistrat, novembre 1993).

Il est possible qu'un des noeuds de la question se situe ici. Revendiquer l'application de la sharî' $a$ islamique pourrait en effet bien traduire la volonté de transformer ce qui a le statut de norme (c'est-à-dire de rationalisation a posteriori, de justification, de répertoire d'argumentation) — et correspond donc à une anticipation de ce qui est socialement reconnu et souhaitable et à son imputation sur ce même social - en un ensemble de règles prescriptives et proscriptives (non plus explicatives). Il y aurait, en quelque sorte, inversion structurelle : d'un «ordre culturel» traduit et manipulé par la norme, on passerait à un «ordre juridique» agissant sur la culture et lui assignant ses normes légitimes. Ce passage s'effectue probablement par une opération de «substantialisation» de la norme. Ceci n'est toutefois possible qu'à la seule condition que le répertoire normatif de départ puisse être investi de qualités régulatoires, ce qui tient généralement au fait qu'historiquement et idéologiquement il ait effectivement fonctionné de la sorte et/ou ait été considéré comme tel. Tel est assurément le cas de la sharî' $a$. Cette condition n'est cependant pas à elle seule suffisante. Elle doit être couplée à des conditions de nature plus politique poussant certains acteurs à souhaiter investir le répertoire normatif de ces qualités régulatoires, et cela implique de réintroduire la notion bourdieusienne de champ (juridique et surtout politique), à la fois «champ de forces» et «champ de luttes». L'enjeu du passage du normatif au régulatoire est, avant tout, un enjeu propre au champ en question dont les composantes sont instrumentalisées en fonction des rapports de force. Mais, quoiqu'il en soit des oppositions entre les différents acteurs de ce champ, ils lui appartiennent tous, avec leurs habitus, leurs «principes générateurs de pratiques», et donc avec leur langage et ce qu'il suppose de schèmes classificatoires communs.

Reprenons notre raisonnement. Le droit (et le discours qui porte sur lui) est communication. En ce sens, il s'agit d'un système symbolique, produit d'interactions et d'échanges, qui médiatise le rapport au monde. Dans la mesure où il est porteur d'enjeux, il constitue un champ, à la fois structuré et récursivement structurant. Comme le propre du droit semble résider dans sa double nature normative et régulatoire, il est aussi bien justification, c'est-à-dire rationalisation a posteriori, que prescription. La double nature justificative et prescriptive du droit opère toutefois toujours sur la base d'une représentation du social, d'une culture, que l'on prétend traduire ou produire. Ces 
deux dimensions ne sont d'ailleurs pas contradictoires, dès lors que la production de la règle prétend souvent se justifier en tant que traduction de la norme. Ce qu'il importe de relever, c'est qu'aussi bien la justification que la réglementation fonctionnent au départ de classifications et de catégories. La norme prétend justifier les habitus, qui euxmêmes sont «des principes de classement, des principes de hiérarchisation, des principes de division qui sont aussi des principes de vision, bref tout ce qui permet à chacun de nous de distinguer des choses que d'autres confondent, d'opérer une diacrisis, un jugement qui sépare»21. Quant à la règle, elle prétend codifier les comportements et, ce faisant, elle fait «passer à l'état objectivé» 22 les classifications et catégories qu'elle prétend déduire du social et qu'en fait elle lui impute. On perçoit le passage qui s'opère quand, de la norme à la règle, on passe de «schèmes maitrisés à l'état pratique» à la «constitution d'une grammaire», c'est-à-dire à une «mise en ordre symbolique», avec ce que cela a pour effets de reproduction/imitation, d'officialisation/homologation et de formalisation ${ }^{23}$. Ce dernier effet est particulièrement important, dans la mesure où il fait passer d'une situation de forte indétermination à une situation claire faite de frontières nettes et tranchées ${ }^{24}$. Mais, quoiqu'il en soit du degré d'indétermination, il est toujours question de catégorisation. Fonctionnant sur la base de schèmes sociaux et culturels, cette catégorisation n'opère pas seulement à l'égard du réel tel qu'appréhendé par le groupe social. Elle exerce un impact sur la définition que ce groupe se donne de luimême, sur ce qui relève de l'«ingroup» et de l'«outgroup». Elle agit donc sur la formulation de l'identité et de l'altérité.

\section{Droit et typification}

L'assignation catégorielle est apparue comme un des modes centraux de fonctionnement du droit. Le propre de pareille fonction réside dans la délimitation d'espaces. En d'autres termes, il apparaît que le droit procède à des définitions assignant à des êtres, des objets ou des situations un espace opératoire distinctif. On observe donc nécessairement la constitution de lieux dotés d'un en-dedans et d'un en-dehors. L'appartenance ou la non-appartenance à ces lieux conditionne le type de rapport à l'autorité et au droit que l'acteur peut avoir. Il est dès lors pertinent de parler des lieux du droit. Ces lieux sont assurément multiples, se recoupent en tout ou en partie et recouvrent des êtres, des objets et des situations en fonction du capital juridique qu'ils détiennent, c'est-à-dire de la position qu'ils occupent à l'intérieur des espaces ainsi

21 Bourdieu P. 1986, «Habitus, code et codification». Actes de la Recherche en Sciences Sociales. 64: 41.

$22 \quad$ Ibid.

$23 \quad I d .: 42$.

24 On peut noter, à cet égard, que le passage de la norme à la règle conduit à une substantialisation de ce qui jusqu'alors relevait davantage du sens commun. Tel est l'une des conséquences majeures de cette «force de la forme» dont parle Bourdieu à propos du droit. Pour prendre un exemple, on peut en effet estimer que la référence à la sharî́a dans la Constitution égyptienne n'était d'abord que la référence à une norme - et n'imposait dès lors qu'une démarche de justification -, mais que la simple formalisation du référent qu'elle constituait a eu pour effet d'enclencher un processus visant à substantialiser ce référent et donc à lui donner le statut de règle. La jurisprudence récente de la Haute Cour Constitutionnelle de même que la condamnation en appel de Nasr Hâmid Abû Zayd sont là pour attester de cet effet de codification faisant passer du statut de norme au statut de règle. 
constitués. La définition de lieux spécifiques se traduit par l'instauration de limites, de frontières, par ce que l'on pourrait appeler un processus de liminarisation. En ce sens, le droit participe à l'affirmation identitaire, sans pour autant que celle-ci ne puisse être envisagée autrement qu'en termes interactionnistes et non substantiels.

Le questionnement dont relève cette proposition théorique procède d'abord de l'affirmation par les différentes personnes interrogées de ce que l'islam se démarque radicalement des autres cultures juridiques ou, du moins, que l'islam se distingue du fait même qu'il est doté d'une culture juridique aux principes fondamentaux caractéristiques.

«Nous appartenons à ceux qui disent que toute civilisation a un projet civilisationnel. Ce qui contribue au développement de l'humanité, c'est la compétition pacifique des projets civilisationnels. C'est ce qui fait que notre position est différente de la position de l'Occident. L'Occident considère que ce qui contribue au progrès de l'humanité, c'est l'emprise par la force dans la mesure où cela conduit au projet civilisationnel, tandis que nous considérons que ce qui contribue au progrès, c'est la compétition pacifique des projets civilisationnels. Il n'est pas possible que la Communauté de l'islam, qui compte plusieurs centaines de millions de membres, propose un projet civilisationnel qui soit déconnecté de sa Loi islamique. Ce ne serait pas son projet» (entretien avec AW, avocat et ancien magistrat, juin 1994).

Approcher le droit en termes de lieux, c'est, surtout dans le contexte qui nous occupe, l'envisager au-travers des représentations que les différents acteurs peuvent avoir de ces lieux et de leur délimitation. En quelque sorte donc, le droit serait métaphoriquement au social ce que la cartographie est à l'espace : une carte dont l'établissement est nécessairement une déformation. Cette proposition, avancée par Boaventura De Sousa Santos ${ }^{25}$, mérite qu'on s'y attarde. Si l'on admet le caractère aporétique d'une analyse de l'effectivité du droit, c'est-à-dire de sa correspondance avec le social ${ }^{26}$, c'est sans doute pour considérer que la représentation du social qu'il propose ne peut être que déformée et que la forme même qu'il prétend lui donner répond à des enjeux et des finalités d'ordre politique et social.

Un des acquis majeurs du pluralisme juridique est d'avoir définitivement mis fin à l'assimilation du droit à l'État et à son territoire. La diversité du droit dépasse les différents cadres nationaux. La multiplicité juridique traduit la multiplicité des espaces sociaux (nationaux, internationaux, communautaires, ethniques, corporatistes, etc., tout ce que Moore qualifie de champ social partiellement autonome) qui sont en interaction constante. Chaque espace, chaque champ, est le lieu d'une compétition pour la détention d'un capital juridique spécifique, qui détermine l'initiative d'un type particulier d'action et le recours à un univers symbolique particulier ${ }^{27}$. Le droit devient, dans ce cadre, un mode de manipulation du social et non pas sa simple traduction. L'identification des différents droits et de leurs niveaux suppose dès lors de tenir compte des règles de manipulation dont ils sont dotés et par lesquelles ils procèdent aux classifications et

25 De Sousa Santos B. 1987, «Law : A Map of Misreading. Toward a Postmodern Conception of Law». Journal of Law and Society. 14/3: 279-302, traduit sous le titre «Droit : une carte de la lecture déformée. Pour une conception post-moderne du droit». Droit et société. 10: 363-390.

26 Griffiths J. 1990, «Legal Pluralism and the Social Working of Law». (non publié) ; Dupret B. 1995, «La sharî̀a comme référent législatif : du droit positif à l'anthropologie du droit». Revue interdisciplinaire d'études juridiques. 34: 99-153.

27 De Sousa Santos, op. cit;: 286. 
délimitations qui leur sont consubstantielles. Si l'on respecte la métaphore de la cartographie, ces règles sont de trois types : l'échelle, la projection et la symbolisation.

L'inclusion d'un être, d'une chose ou d'une situation dans un espace juridique est souvent multiple et la lecture que l'on peut avoir du type de relations juridiques qui l'affectent dépend étroitement de l'échelle juridique pour laquelle on opte (locale, nationale, internationale, si l'on reprend la typologie de De Sousa Santos). Cela signifie qu'un même objet peut recevoir différentes qualifications juridiques. Par ailleurs, les différents espaces juridiques fonctionnent simultanément et interagissent (interlégalité, interdroit) et c'est dans cette interaction que se définissent leurs frontières et les seuils régulatoires leur donnant accès. Ces échelles juridiques ne doivent pas être prises au seul sens objectif d'une coexistence de différents ordres normatifs au spectre variable. On peut également les considérer au sens subjectif de la représentation que les acteurs du droit se font de leur position au sein des espaces juridiques et des manipulations qui sont offertes dans ce cadre. L'acteur peut en effet jouer sur les échelles et les seuils régulatoires, participant ainsi à la définition de la légalité pertinente à ses yeux et à l'inclusion dans cette légalité d'êtres, d'objets ou de situations. A cet effet, il prend en considération différents indices et critères d'identification, de discrimination et d'évaluation. Le choix de l'échelle conduit ici l'acteur à méconnaître ou à mettre l'accent sur tel ou tel signe distinctif. La projection est, pour sa part, la procédure par laquelle l'ordre légal définit les limites de ses opérations et s'organise à l'intérieur de ces limites $^{28}$. Différentes projections peuvent être réalisées, qui mettent l'accent sur la formulation spécifique d'intérêts différents (les objets sociaux sont donc doublement susceptibles de donner naissance à des objets juridiques différents). En fonction du type de projection, êtres, choses et situations sont dotés d'un capital juridique différent et d'une position plus ou moins centrale ou périphérique au sein de l'espace juridique considéré. Ceci détermine bien évidemment des relations de pouvoir qui prennent la forme de méthodes interprétatives et de configurations idéologiques. Ces dernières ne se donnent pas pour telles et tendent à effacer leurs spécificités pour être plus facilement exportables à la périphérie à laquelle elles prétendent s'appliquer de manière égale ${ }^{29}$. Ici encore, il ne s'agit pas de considérer l'effet de projection sous le seul angle objectif d'ordres normatifs imposant une structure sociale inégalitaire et différenciée, mais également sous l'angle subjectif de représentations du droit procédant au centre ou en périphérie des différents espaces juridiques et développant de ce fait des mécanismes d'acceptation ou de rejet des ordres normatifs imposés et donc, si l'on reprend la distinction de Michel de Certeau, des stratégies ou des tactiques selon qu'ils sont au coeur de l'ordre dominant ou qu'ils doivent s'en jouer ${ }^{30}$. Il faut enfin noter que différents types de symbolisation peuvent correspondre aux différents ordres juridiques. Cette symbolisation est étroitement fonction de l'échelle juridique et du type de projection. Bien entendu, les différents styles, au même titre que les différents ordres juridiques, peuvent coexister et les acteurs du droit peuvent être tentés de passer d'un ordre à l'autre

\footnotetext{
$28 \quad$ Id.: 291.

29 Il y a là une autre voie par laquelle le profit d'universalisation trouve à se réaliser.

30 Certeau (de) M. 1980, L'invention du quotidien. 1: Arts de faire. Paris: Union générale d'éditions ; Laurent P.-J., non publié, «L'art de la ruse et le don à un étranger. Esquisse empirique d'une théorie anthropologique de la coopération au développement». Thèse de doctorat, Université catholique de Louvain.
} 
et donc d'utiliser alternativement les différentes rhétoriques et symboliques en fonction du contexte qui semble s'imposer, des intérêts qu'il s'agit de promouvoir et des rapports (de force) dans lesquels cela s'inscrit. C'est en ce sens que l'on fait référence à des répertoires juridiques à la disposition des acteurs du droit.

Ce que nous enseigne la métaphore cartographique est double. D'une part, on constate la multiplicité des lieux du droit, avec son corollaire d'interlégalité. Le droit s'inscrit dans un espace et les êtres, choses et situations s'inscrivent dans des espaces juridiques éventuellement concurrents et concomitants. Ces ordres normatifs, à l'échelle variable et à la symbolique différenciée, loin d'être homogènes, renferment des déséquilibres entre un centre et une périphérie imprégnée d'éléments allogènes et particulièrement perméable à l'incidence de facteurs exogènes. D'autre part, cette situation fait que les acteurs du droit ont tendance à jouer de la pluralité des espaces, souvent en fonction de la position centrale (stratégies) ou périphérique (tactiques) qu'ils y occupent et en manipulant les symboliques et les rhétoriques à leur disposition.

De manière générale, il semble évident qu'au moins une certaine forme légale s'est progressivement et globalement imposée. Ce constat est en tout cas valable pour l'Égypte et, plus largement, pour le monde arabe. Ce transfert de technologie juridique permet de situer le droit égyptien et le discours de ses acteurs à la périphérie d'un espace juridique plus global - et ceci sans préjuger d'autres espaces juridiques que la notion de «droit égyptien» recouvrirait également. Dans cet espace global, doté de son langage et de sa symbolique propres, le capital juridique est inégalement réparti, non seulement entre schématiquement l'Occident et l'Égypte, mais aussi, en Égypte même, entre les différents pôles du champ juridique et entre ses différents acteurs. Mais, précisément, la notion de «droit égyptien» recouvre d'autres espaces juridiques que le seul espace global. Elle constitue donc, dans son essence même, une situation d'interlégalité où les différents espaces légaux, parce qu'ils ne sont pas synchroniques, produisent «des mélanges inégaux et instables de codes légaux (codes dans un sens sémiotique)» ${ }^{31}$. Le droit égyptien ne procède pas plus du seul espace légal «global» qu'il ne procède du seul espace légal «indigène». Il procède, au contraire, du couplage de ces deux espaces qu'il greffe l'un à l'autre.

\section{Droit et identification}

Une observation attentive de la scène sociale, politique et juridique égyptienne permettrait d'aisément identifier les modes de solidarisation et de polarisation que la transplantation de la technologie juridique occidentale a pu générer. Il reste que ces différentes réactions n'opèrent que rarement le rejet du droit transplanté au profit du seul ordre normatif considéré comme indigène. Au contraire, un certain type de juridicité semble avoir bien pris racine. Il reste à comprendre comment cela a pu être rendu possible. A cet égard, l'analyse de Massaji Chiba sur le postulat d'identité du droit indigène et sur sa fonction dans la transplantation juridique est de première importance $^{32}$. Chiba fait d'abord le constat qu'un certain nombre de principes de droit

31 De Sousa Santos, op. cit.: 298.

32 Chiba M. 1986, «The Identity Postulate of Indigenous Law and its Function in Legal Transplantation». In Sack P. and Minchin E. (eds.), Legal Pluralism. Proceedings of the Camberra Law Workshop VII. Camberra: Australian National University. 
positif ne peuvent être compris et ne trouvent pas à s'appliquer (du moins de manière autonome), dans les traditions juridiques non occidentales, sans qu'il ne soit fait recours à ce qu'il appelle des «compléments fonctionnels». Pour pouvoir opérer, ces compléments fonctionnels doivent prendre la forme d'un cadre de référence permettant à certaines normes socio-culturelles de fonctionner de pair avec le cadre légal établi (droit positif). Ceci implique, naturellement, d'avoir sur le droit un regard anthropologique reconnaissant la pertinence de la théorie du pluralisme juridique. A ce stade, Chiba construit son analyse sur trois dichotomies : droit officiel - droit non officiel ; règle légale - postulat juridique (ou règle positive - valeur «postulative»); droit indigène droit transplanté33. Dans un contexte de transplantation juridique, le droit non officiel (généralement le droit indigène) devient le véhicule par lequel, sous forme de postulat juridique (ou éventuellement de règle juridique), les compléments fonctionnels trouvent à remplir leur rôle. Les postulats juridiques proposent ainsi la traduction juridique des compléments fonctionnels dont la formulation est d'ordre plutôt socio-culturel. Il faut faire le constat de ce que l'exportation d'une technologie juridique étrangère ne constitue rien moins qu'une crise culturelle dans le chef de la population réceptrice, dans la mesure où son identité culturelle (et/ou les représentations qu'elle s'en fait) peut s'en trouver sérieusement perturbée ou même oblitérée ${ }^{34}$. C'est pour cela que le choix d'adopter le droit étranger (ou certaines de ses caractéristiques), plus ou moins reformulé, doit se faire de manière à permettre à l'identité culturelle et à ce que la population concernée s'en représente de se perpétuer. Il en va de même du choix des éléments de droit indigène, plus ou moins reformulés, devant être intégrés au nouveau système ${ }^{35}$. La notion de «postulat d'identité du droit indigène» vise précisément le postulat juridique de base permettant à un groupe de maintenir, dans le droit, son identité culturelle et ce qu'il s'en représente.

L'existence de postulats juridiques, en ce compris les postulats d'identité, est attestée dans l'ensemble des traditions juridiques. Il ne s'agit de rien d'autre que ce que précédemment on appelait standard juridique ${ }^{36}$. On remarquait, dans ce cadre, que le recours au standard juridique relève d'un passage de la norme entendue au sens statistique (ou ce que l'acteur considère relever de la norme statistique) à la norme entendue au sens juridique et contraignant. Si l'on reprend la distinction de Carol Greenhouse (cf. supra), on sera enclin à dire que les postulats juridiques (dont celui d'identité) appartiennent à l'ordre de la justification, c'est-à-dire de la norme culturelle, davantage qu'à l'ordre de la prescription, c'est-à-dire de la règle de droit à proprement parler. Tel est probablement le cas de la sharî'a dans le contexte égyptien qui nous occupe. C'est en ce sens qu'en tout cas nous interprétons le petit extrait que voici.

«Q : Quand j'ai rencontré BI, il m'a dit que ce qui manquait, ce n'était pas les textes, mais la référence, le texte de référence. Les textes appliquent la sharî‘ $a$, mais on ne trouve pas cette référence globale.

«R : Oui, c'est vrai. D'où vient ce cadre référentiel ? Il faudrait que ce soit l'interprétation des textes et leur application en faisant référence aux fondements de l'islam. Si nous trouvions ce cadre référentiel

\footnotetext{
$33 \quad$ Id.: 39-40.

$34 \quad$ Id.: $44-45$.

$35 \quad$ Ibid.

36 Dupret B. 1995c, «Le juge et le jeu de la normalisation islamique du droit positif». Droit et Cultures. 30: 47-64.
} 
présent, $90 \%$ de nos difficultés seraient résolus» (entretien avec AW, avocat et ancien magistrat, juin 1994).

Le postulat d'identité se caractérise par un contenu éminemment variable : il a une fonction plus qu'une substance. Ceci seul permet d'ailleurs d'expliquer qu'il serve aussi bien à invalider le droit transplanté qu'à favoriser son accomodation dans un contexte changeant ${ }^{37}$. Dans les deux cas, son efficacité n'est garantie que par sa plasticité. Celleci procède de deux caractéristiques qui nous intéressent plus particulièrement, dans le fil de notre raisonnement. D'une part, le postulat tend à circonscrire le ressort d'une collectivité et donc à déterminer les critères d'appartenance à celle-ci. D'autre part, il tend à symboliser un système de valeurs particulier, donnant ainsi aux membres de la collectivité le référent de leur identité et de leur appartenance ${ }^{38}$. On en revient ainsi à l'idée d'un droit participant à la définition de l'identité du groupe, ce qui n'est pas sans soulever une série de questions.

Le constat d'une impossible approche de l'identité en termes substantiels a été établi. On a dit précédemment qu'il fallait entendre la problématique identitaire en termes de mécanisme menant tout groupe social à chercher un ancrage dans des référents perçus comme authentiques, alors même qu'ils sont en constante recomposition. Cela suppose avant tout d'en comprendre les modes de fonctionnement. Dès lors qu'on s'interroge sur la fonction du droit dans le processus d'affirmation identitaire, on peut s'en référer aux théories de l'ethnicité et, plus particulièrement, à la théorie interactionniste telle qu'elle s'est développée depuis l'article fondateur de Fredrik Barth ${ }^{39}$. François Ireton définit l'identité collective comme le «produit symbolique d'une (re)construction sociale continue de différences culturelles opérant l'assignation d'individus à un ensemble social» ${ }^{40}$. Cela souligne le caractère symbolique de l'identité, qui est l'affirmation d'une appartenance et d'une différence exprimée au départ de biens culturels sélectionnés et formulés pour le signifier ou encore, pour reprendre Drummond, «un ensemble d'idées contraignantes sur la distinctivité entre soi et les autres, qui fournit une base pour l'action et l'interprétation des actions d'autrui» ${ }^{41}$. Si l'identité est le produit d'un travail de symbolisation, elle n'est donc jamais objective. Il n'y a pas d'«identité en soi», mais uniquement une production et une reproduction symbolique ${ }^{42}$ que seule l'interaction des groupes permet d'expliquer. L'un des apports majeurs de Barth consiste précisément à avoir démontré que «l'ethnie est avant tout une catégorie d'ascription, dont la continuité dépend d'une frontière et donc d'une codification constamment renouvelée des

37 Les acteurs du droit peuvent avoir intérêt à jouer sur le mode de l'accommodation comme sur celui de l'invalidation. En jouant sur les registres et les répertoires, ils peuvent produire, au niveau discursif à tout le moins, aussi bien un effet de couplage qu'un effet de découplage des différents espaces légaux.

38 Chiba op. cit;: 41.

39 Barth F. 1969, Ethnic Groups and Boundaries. The social organisation of culture difference. London: George Allen \& Unwin (traduction in Poutignat et Streiff-Fenart 1995, «Les groupes ethniques et leurs frontières»).

40 Ireton F., non publié, «A propos de la (re)production d'une identité "sa'idi"».

41 Drummond L. 1980, «The Cultural Continuum : A Theory of Intersystems». Man. 15/2:

368, cité par Poutignat P. et Streiff-Fenart J. 1995, Théories de l'ethnicité, suivi de «Les groupes ethniques et leurs frontières», de Fredrik Barth. Paris: PUF, 121.

42 Ireton, op. cit. 
différences culturelles entre groupes voisins»43. Cela signifie que «lidentité collective n'existe que par sa frontière et que ce qu'elle enclôt n'est pas pertinent dans la détermination de son existence» ${ }^{44}$. L'identité est performative ${ }^{45}$, en ce sens qu'elle n'a d'existence que dans son affirmation. Quant à la frontière, qui n'est que «purement différentielle», elle se construit «à partir d'éléments, de «traits» culturels «objectifs» (matériels et/ou symboliques) potentiellement discriminants que les agents en interaction «choisissent» et érigent en éléments de différentiation, c'est-à-dire en marques identitaires ${ }^{46}$.

En résumé, l'identité collective est cette attribution catégorielle qui classe les personnes et les groupes en fonction de leur appartenance culturelle supposée et que l'interaction sociale valide en faisant ressortir les signes culturels socialement différenciateurs ${ }^{47}$. Pareille définition permet d'identifier une série de problématiques fondamentales : attribution catégorielle, dichotomisation Nous/Eux, fixation des symboles identitaires, saillance des traits distinctifs (ibid.). L'analyse du droit, de ses répertoires et des représentations que les différents acteurs en ont permet de souligner à quel point la norme (particulièrement juridique) en constitue un élément central. Un nouvel extrait d'entretien devrait permettre de s'en convaincre.

«(La Communauté de l'islam) pourrait présenter un projet occidental transformé ou un projet japonais transformé ou un projet asiatique transformé, mais si un projet quelconque est appelé «projet civilisationnel islamique», cela signifie que le projet constitue une part fondamentale et une composante principale de l'islam, construit sur un régime juridique fondé sur la sharî'a islamique. Un élément de principe, c'est que cette Loi islamique représente l'une des faces de notre foi et que nous éprouvons à son égard un besoin comparable à la soif pour l'eau ou à la faim de nourriture. Elle constitue la colonne vertébrale du régime civilisationnel islamique. Si la colonne vertébrale de ce régime vient à vaciller, c'est la civilisation islamique qui disparaît et devient une image transformée de la civilisation occidentale, bouddhiste ou autre. Personne au monde n'a le droit d'interdire à une des communautés de fonder son régime juridique, éducatif et culturel sur son héritage (turâth). Il n'existe pas une communauté au monde qui se soit débarrassé de son héritage. Ce qui s'est produit dans notre pays, c'est une colonisation qui, depuis cent ans jusqu'à maintenant, a supprimé les lois inspirées par la sharî'a islamique. Dans mon livre sur le système pénal islamique, j'ai écrit des choses sur l'Égypte et, en particulier, sur l'effet de la colonisation britannique. Le colonisateur a éduqué des générations d'intellectuels très brillants, mais qui ne connaissaient que la loi du colonisateur et sa civilisation, au point de dire qu'il faut que nous

43 Bonte P. et Izard M. 1991, Dictionnaire de l'Ethnologie et de l'Anthropologie. Paris: PUF:

243.

44 Ireton, op. cit.

45 Ferrié J.-N., à paraître, «Qu'est-ce qu'un copte selon des musulmans ? Construction de la frontière et typification de soi».

46 Ireton, op. cit. On notera l'intérêt de la démarche «à la fois interactionniste et culturaliste» d'Eriksen (Eriksen T. H. 1991, «The Cultural Context of Ethnic Differences». Man. 26/1: 127-144). «Interactionniste, puisque les visions du monde particulières que les individus engagent dans les rencontres interculturelles n'existent pas indépendamment des situations d'interaction au cours desquelles les acteurs les mettent en oeuvre en se proposant mutuellement des jeux de langage (cf. Wittgenstein : «un champ intersubjectif lié à un contexte particulier et reproduit par les individus dans l'interaction»). Culturaliste, puisque ce que les acteurs engagent dans les jeux de langage, ce sont les définitions respectives qu'ils ont des structures de signification du monde pertinentes, c'est-à-dire des cultures entendues dans un sens proche de celui que Geertz donne à ce terme, comme des contextes permettant l'intelligibilité des situations et des événements» (Poutignat et Streiff-Fenart op. cit.: 122). 47 Cf. Poutignat et Streiff-Fenart, op. cit.: 154. 
progressions pour vivre comme vivent les gens de l'Ouest de la mer Méditerranée, que nous parlions comme ils parlent, que nous nous habillions comme ils s'habillent, que nous écrivions de gauche à droite comme ils écrivent. Ce propos était quelque peu sarcastique ou ironique, dans la mesure où, si tu veux humilier quelqu'un, la meilleure chose à faire, c'est de lui dire de se débarrasser de son histoire et de son héritage. Cette conception ironique a encouragé les principaux penseurs islamiques à réagir. Notre indépendance, dans les années cinquante, précédée de peu par l'indépendance syrienne et libanaise et suivie peu après par le Nord de l'Afrique et le reste des pays colonisés, n'a pas changé notre situation juridique d'un pouce, au contraire. Elle a ancré la conformisation juridique sur l'Occident. Les plus grands hommes de loi dans notre pays, comme Sanhûrî, Khashaba, Hudaybî, Abû Stît ou encore le mufti alJazâyirlî, qui étaient les autorités de la justice d'appel à l'époque, ont écrit des livres pour s'opposer à la codification civile qui avait emprunté ses dispositions à l'Occident et ont dit qu'il fallait emprunter nos dispositions à la sharî'a islamique. Ceci fut proposé au Sénat (Majlis al-shuyûkh) et on dispose d'un volume entier des minutes consacrées au droit civil. On y trouve des démonstrations complètes à ce sujet, mais ce fut en vain, parce que le colonisateur imposa sa loi dont hérita un membre de notre communauté qui croyait davantage en leur civilisation qu'en la nôtre. Tel est le conflit aujourd'hui. Il réside dans le fait que c'est notre droit, en tant que communauté qui a une histoire et un héritage, d'être gouvernés et éduqués conformément à notre histoire et à notre héritage. Or nous ne sommes pas gouvernés et éduqués conformément à cela. Première atteinte. La deuxième catastrophe, c'est que le leadership politique qui contrôle la conduite des affaires dans notre pays ne pense pas que l'islam constitue un projet civilisationnel indépendant. De plus, l'Occident continuera à être incapable de développer un projet humain; il sera maître au point de vue technologique et matériel, il inventera la sixième, septième, dixième génération informatique, il ira sur la lune ou fabriquera n'importe quelle technologie, mais il ne constuira rien d'humain, parce que la lutte qui contribue à l'amélioration du projet humain est réduite à néant. Personne ne lutte avec lui sur le plan humain, personne n'oppose les valeurs des religions à cet Occident. Il y a, en Occident, des gens religieux par millions, mais ils sont individuellement religieux et ne répercutent pas leur religiosité sur le projet politique, économique ou social. C'est cela que, nous autres, nous proposons. Nous disons que la compétition entre le projet islamique et le projet occidental conduit à un mieux pour l'humanité. Les musulmans, en effet, ont tiré parti des bienfaits de l'Occident au point de vue civilisationnel comme l'Occident a tiré parti de l'islam auparavant — il n'est pas besoin de le mentionner. La poursuite de la compétition civilisationnelle contribuera donc au bien de l'humanité. Voilà la première question centrale de cette problématique de l'application de la sharî‘ $a$ islamique» (entretien avec AW, avocat et ancien magistrat, juin 1994)

Le droit, dans son oeuvre de typification (fixation identitaire), fonctionne par affirmation de continuités et de discontinuités : continuités historiques au travers de réinterprétations ${ }^{48}$, discontinuités culturelles au travers d'un processus de «liminarisation» (constitution de frontières définissant le rattachement identitaire). Le discours juridique et l'emphase donnée à l'un ou l'autre répertoire juridique traduisent cette fonction typificatrice de la norme (de droit). Comme dans la parabole du bouc émissaire $^{49}$ que rapporte François Ost, à la suite de Michel Serres, le droit sert à la construction d'une unité fondée «sur une opération de division et une pratique d'exclusion ${ }^{50}$. La langue juridique et le discours sur le droit constituent une symbolique

48 «Processus par lequel de vieilles significations sont imputées à de nouveaux éléments ou par lesquelles de nouvelles valeurs changent la signification d'anciennes formes. Cela opère à l'intérieur, de génération en génération, par l'intégration à une culture réceptrice d'un élément emprunté» (Herskovits M. 1948, Man and His Works. New York: Alfred A. Knopf: 553, cité par Hamer J. H. 1994, «Identity, Process, and Reinterpretation. The Past Made Present and the Present Made Past». Anthropos. 89: 182).

49 La parabole du bouc émissaire «évoque une frontière fermée ; en quête de son homogénéité ou de sa pureté, le groupe s'invente un principe de division (la cité/le désert) et se forge un critère d'exclusion (les innocents/le coupable), le tout doublé d'une logique économique-utilitariste imparable (un sacrifié/quatre-vingt-dix-neuf sauvés)» (Ost, à paraître). 50 Ost F., à paraître, «Les frontières de la juridicité : dialectique ou autopoièse ?». Ceci, sans préjuger de la possibilité d'une unité fondée sur un principe d'inclusion et une pratique 
facilitant la fixation et la polarisation des identités au départ de critères de pertinence faisant valoir certains traits distinctifs et en occultant d'autres. Quant au comportement des acteurs, il vise avant tout à produire les apparences de la conformité aux règles du groupe, «lors même que leur pratique est en contradiction avec la règle ou qu'elle n'a pas pour principe l'obéissance pure à la règle» ${ }^{51}$, montrant ainsi que ce qui compte, avant tout, c'est l'affirmation ostensible d'appartenance au groupe, non l'adoption des pratiques qui lui sont substantiellement propres.

\section{Bibliographie}

Assier-Andrieu L. 1987, «Le juridique des anthropologues». Droit et Société. 5: 89107

Bailey F. G. 1973, Debate and Compromise : The politics of innovation. Totowa: Rowman \& Littlefield

Barth F. 1969, Ethnic Groups and Boundaries. The social organisation of culture difference. London: George Allen \& Unwin (traduction in Poutignat et Streiff-Fenart 1995, «Les groupes ethniques et leurs frontières»)

Berger P. L. and Luckmann T. 1966, The Social Construction of Reality : A Treatise in the Sociology of Knowledge. New York: Doubleday (traduction 1989, La construction sociale de la réalité. Paris: Méridien Klincksieck)

Bonte P. et Izard M. 1991, Dictionnaire de l'Ethnologie et de l'Anthropologie. Paris: PUF

Bourdieu P. 1986, «Habitus, code et codification». Actes de la Recherche en Sciences Sociales. 64: 40-44

Bourdieu P. 1994, Raisons pratiques. Sur la théorie de l'action. Paris: Seuil

Certeau (de) M. 1980, L'invention du quotidien. 1: Arts de faire. Paris: Union générale d'éditions

Chiba M. 1986, «The Identity Postulate of Indigenous Law and its Function in Legal Transplantation». In Sack P. and Minchin E. (eds.), Legal Pluralism. Proceedings of the Camberra Law Workshop VII. Camberra: Australian National University

Cohen A. P. and Comaroff J. L. 1976, «The management of meaning: on the phenomenology of political transactions». In Kapferer B. (ed.), Transaction and meaning. Philadelphia: Institute for the Study of Human Issues

Crespi F. 1993, v ${ }^{\circ}$ «Action (sociologie de l')». In Arnaud A.-J. (sous la dir.), Dictionnaire encyclopédique de théorie et de sociologie du droit. Paris: LGDJ

De Sousa Santos B. 1987, «Law : A Map of Misreading. Toward a Postmodern Conception of Law». Journal of Law and Society. 14/3: 279-302, traduit sous le titre «Droit : une carte de la lecture déformée. Pour une conception post-moderne du droit». Droit et société. 10: 363-390

Drummond L. 1980, «The Cultural Continuum : A Theory of Intersystems». Man. 15/2: $352-374$

d'intégration que symbolise la parabole symétrique de la brebis perdue. Celle-ci «évoque une frontière ouverte ; paissant de-ci de-là, le groupe pastoral ne s'enferme pas dans une enceinte : son unité est réelle pourtant, dont la garde est confiée au berger. Chaque bête à ses yeux a valeur d'ensemble, puisque le voilà prêt à en exposer quatre-vingt-dix-neuf pour en ramener une seule» (id.).

51 Bourdieu P. 1994, Raisons pratiques. Sur la théorie de l'action. Paris: Seuil: 239. 
Dupret B., à paraître, «Solidarité de Logiques idéelles sans consensus politique. Représentations des répertoires juridiques positif et islamique parmi les avocats de la tendance islamique égyptienne». Journal of Legal Pluralism

Dupret B. 1995a, «La sharî'a comme référent législatif: du droit positif à l'anthropologie du droit». Revue interdisciplinaire d'études juridiques. 34: 99-153

Dupret B. 1995b, «Le juge et le jeu de la normalisation islamique du droit positif». Droit et Cultures. 30: 47-64

Durant G. 1992, Les structures anthropologiques de l'imaginaire. Paris: Dunod

Eriksen T. H. 1991, «The Cultural Context of Ethnic Differences». Man. 26/1: 127144

Ferrié J.-N., à paraître, «Qu'est-ce qu'un copte selon des musulmans ? Construction de la frontière et typification de soi»

Ferrié J.-N., Boëtsch G. et Ouafik A. 1994, «"Vécu juridique", norme et sens de la justice : à propos de l'avortement au Maroc». Droit et société. 28: 677-690

Ferry J.-M. 1991, Les puissances de l'expérience. Paris: Cerf

Greenhouse C. J. 1982, «Looking at culture, looking for rules». Man. 17: 58-73

Griffiths J., non publié, «Legal Pluralism and the Social Working of Law»

Habermas J. 1985a, Le discours philosophique de la modernité. Douze conférences. Paris: Gallimard

Habermas J. 1985b, Morale et communication. Paris: Cerf

Habermas J. 1987, Théorie de l'agir communicationnel (2 tomes). Paris: Fayard

Hamer J. H. 1994, «Identity, Process, and Reinterpretation. The Past Made Present and the Present Made Past». Anthropos. 89: 181-190

Herskovits M. 1948, Man and His Works. New York: Alfred A. Knopf

Ireton F. 1995, «A propos de la (re)production d'une identité "sa'idi"», non publié

Kapferer B. (ed.) 1976, Transaction and meaning. Philadelphia: Institute for the Study of Human Issues

Laurent P.-J., non publié, «L'art de la ruse et le don à un étranger. Esquisse empirique d'une théorie anthropologique de la coopération au développement». Thèse, Université catholique de Louvain

Lenoble J. 1994, Droit et communication. Paris: Cerf

Melkevik B. 1990, «Le modèle communicationnel en science juridique : Habermas et le droit». Les Cahiers de Droit. 31/3: 901-915

Moore S. F. 1978, Law as Process : An Anthropological Approach. London, Henley and Boston, Routledge \& Kegan Paul

Ost F. 1987, «Essai de définition et de caractérisation de la validité juridique». In Rigaux F. et Haarscher G. (sous la dir.), Droit et pouvoir. t. I - La validité. Bruxelles: Story-Scientia

Ost F., à paraître, «Les frontières de la juridicité : dialectique ou autopoièse ?»

Paine R. 1976, «Two modes of exchange and mediation». In Kapferer B. (ed.), Transaction and meaning. Philadelphia: Institute for the Study of Human Issues

Herskowits M. 1948, Man and His Works. New York: Alfred A. Knopf

Poutignat P. et Streiff-Fenart J. 1995, Théories de l'ethnicité, suivi de «Les groupes ethniques et leurs frontières», de Fredrik Barth. Paris: PUF

Salisbury R. F. 1976, «Transactions or transactors? An economic anthropologist's view». In Kapferer B. (ed.), Transaction and meaning. Philadelphia: Institute for the Study of Human Issues 
Teubner G. 1992, «Pour une épistémologie constructiviste du droit». Annales ESC. 6: $1149-1169$

Teubner G. 1993, Le droit, un système autopoiétique. Paris: PUF

Teubner G. 1994, Droit et réflexivité. L'auto-référence en droit et dans l'organisation. Bruxelles-Paris: Story-Scientia-LGDJ

Verdier R. 1981, «Premières orientations pour une anthropologie du droit». Droit et Cultures. 1: 5-22

Vignaux G. 1979, «Argumentation et discours de la norme». Langages: 53: 67-85

Winkin Y. 1993, $\mathrm{v}^{\circ}$ «Interactionnisme symbolique». In Arnaud A.-J. (sous la dir.), Dictionnaire encyclopédique de théorie et de sociologie du droit. Paris: LGDJ 